\title{
A NEW METHOD OF PERFORMING FIELD TRIALS
}

\author{
BY BERNARDO G. CAPÓ
}

Biometrician

\section{INTRODUCTION}

In a recent article (1) in which the author discussed a new method of interpreting the results of field trials, he presented evidence tending to show that the åssumption of a different effect constant for every pair of adjacent plots of a randomized block field trial led to a greater precision in the reduction of the corresponding data than the assumption of a different effect constant for every different complete block of the experiment. In said article he presented a method which might be used in this connection in order to reduce the calculational work to a minimum for the interpretation of such experiments by use of the proposed new assumption. The work involved in the application of said method to the results of randomized block experiments already performed, however, is greater than the work required for the application of the usual methods of statistical analysis in regard to which said experiments have been designed. On the other hand, the increase in precision obtained by the application of the suggested method seems to the author to warrant fully the increase in such calculational work.

As it was also mentioned in the article referred to, it is possible to reduce the number of operations required for the application of that method to the interpretation of field trials by designing such field trials so as to make use of the proposed method. The purpose of this article is to suggest designs for possible use in this connection as well as to discuss the procedure to be followed in the interpretation of such specially designed experiments.

REQUIREMENTS TO BE FULFILLED BY FIELD TRIALS

Field trials must fulfill certain requirements in order that the application of the proposed method to the interpretation of their results be most economical in time and effort. These requirements are as follows: 1-As many different groups of two treatments each must be made as is possible. 2The experimental field must be divided into blocks of 2 -adjacent plots each, so that the topographies of the two plots in any given block will be as nearly alike as possible. 3-Each group of two treatments must be assigned at random to be tested in one of the 2-plot blocks. The plot of the respective 2-plot block in which one or the other of the two treatments of the group assigned to it will be tested must be also decided at random. 4-The same number of replications of each group of treatments must be used. That is, 
if $n$ treatments are to be tried, there should be $m(n-1)$ replications, where $m$ is any positive integer. Thus, if 6 treatments are to be compared, either 5 or 10 or 15 , etc. replications of the treatments are required.

EXAMPLES OF DESIGNS OF POSSIBLE USE

In order to give illustrations of the possibilities of this type of layout, two examples will be given of possible different designs for a trial in which 5 treatments are repeated 4 times each.

Where 5 treatments, say $A, B, C, D$, and $E$, are to bo repeated 4 times each, the experiment should include 10 groupings as follows: $A B, A C$, $A D, A E, B C, B D, B E, C D, C E$, and $D E$. The 20-plot experimental field is divided into ten 2-plot blocks, and the above treatment pairs are

Diagram I

- Possible layouts of plots

\begin{tabular}{c|c|c|c|c|}
\hline & (a) & (b) \\
\hline $\mathrm{AB}$ & $\mathrm{BC}$ & $\mathrm{AB}$ & $\mathrm{EC}$ & $\mathrm{D}$ \\
\hline $\mathrm{EC}$ & $\mathrm{EA}$ & $\mathrm{ED}$ & $\mathrm{CB}$ & $\mathrm{A}$ \\
\hline $\mathrm{AD}$ & $\mathrm{BE}$ & $\mathrm{AE}$ & $\mathrm{BD}$ & $\mathrm{C}$ \\
\hline $\mathrm{ED}$ & $\mathrm{AC}$ & $\mathrm{CA}$ & $\mathrm{EB}$ & $\mathrm{D}$ \\
\hline $\mathrm{DB}$ & $\mathrm{CD}$ & & \\
\hline
\end{tabular}

- assigned at random to be tested in one or another of the 10 blocks, there being also randomization of the order of the treatments to be tested whin each block. A possible geographical distribution might be as indicated by Diagram I (a).

Another possible arrangement, which lends itself to possible interpretation by either the 2-plot block method or by the ordinary method of analysis for a 4-randomized-block trial, is shown in Diagram I (b).

\section{THEORY OF THE PROCEDURE OF CALCULATION}

As indicated in the article (1) previously referred to, the regression equation to be used in explaining the results of such an experiment would be

$$
Y_{i}=B_{i}+T_{i},
$$

where $Y_{i}$ is the observed effect, say yield, of plot $i, B_{i}$ is the effect constant of the block of which plot $i$ forms part, and $T_{i}$ is the effect constant of the treatment tested in plot $i$. After writing down the respective equation 
for each of the plots used, the problem becomes one of finding the most probable values of the $B_{i}$ 's and the $T_{i}$ 's, and the standard errors of the latter for comparison with one another. It was suggested in said article that a convenient way to do this would be one in which the differences between the corresponding figures of the paired plots were found and then, following the usual methods of regression analysis, determining the most probable values of the $T_{i}$ 's and their standard errors.

Now, if the experiment has been carried out in such a way that every treatment has been thus paired with every other treatment once, it will be found that by the application of the above procedure, the value of each of the $C_{i i}$ 's will come out to be $2(n-1) / n^{2}$ and the value of each of the $C_{i j}$ 's $(i \neq j)$ will come out to be $-2 / n^{2}$ where $n$ is the number of treatments tested.

By knowing then $S a y, S b y, S c y, S d y, S e y$, and $S y y$, and by the proper use of the $C_{i i}$ 's and $C_{i j}$ 's, already known, the values of the treatment effect constants, i.e., $A, B, C, D$, and $E$, may be found together with their standard errors. One may thus test the significance between the estimated difference in effects between any two of the treatments tested.

The theory of the procedure outlined in the last paragraph is so well known to those interested in it, that it does not merit further consideration. It may be worthwhile, however, to derive the relations $C_{i i}=2(n-1) / n^{2}$ and $C_{i j}(i \neq j)=-2 / n^{2}$. This will be done now.

Let it be assumed that $n$ treatments have been tested in a trial of the kind under consideration. Let the effects of the different treatments above or below the mean effect of all treatments be designated by $A, B, C, \cdots, N$, where $N$ is the effect of $n$th treatment. The effect equations of the type

$$
Y_{i}=B_{i}+T_{i}
$$

may then be written for each plot.

Since any given treatment, say $A$, has been replicated $n-1$ times, there will be $n-1$ such equations in which the treatment effect $A$ appears. Also, on account of this, there will be $n-1$ equations formed by finding the differences between the equations corresponding to the treatment $A$ plots and those corresponding to the other plots paired to said treatment $A$ plots. These equations might be written as:

$$
\begin{aligned}
& \pm A \mp B=d_{ \pm A \mp B} \\
& \pm A \mp C=d_{ \pm A \mp C} \\
& \pm A \mp D=d_{ \pm A \mp D} \\
& \cdot \quad \\
& \vdots \\
& \pm A \mp(N-1)=d_{ \pm A \mp(N-1)} \\
& \pm A \mp N=d_{ \pm A \mp N}
\end{aligned}
$$


where the $d$ 's stand for the respective differences between the effects noticed in the $A$ plots as against the effects noticed in the plots paired with the $A$ plots. Since, however, $A, B, C, \cdots$, and $N$ have been assumed to be the effects of the treatments above or below the mean effect of all the treatments,

$$
A+B+C+\cdots+N=0
$$

or

$$
N=-(A+B+C+\cdots+(N-1))^{\circ}
$$

The last of the above equations, therefore, should appear rather as $\pm 2 A \pm B \pm C \pm \cdots \pm(N-1)=d_{ \pm A \mp N}$, due to the relation for $N$ previously pointed out. Further, the effect constant $A$ would also appear in all the equations corresponding to comparisons between plots receiving treatment $N$ and anyone of the other treatments.

In other words, $A$ appears with a coefficient of \pm 1 in the $n-2$ equations corresponding to treatment $A$ comparisons with treatments $B, C, D, \cdots$, $N-1$, and also in the $n-2$ equations corresponding to the comparisons of treatments $B, C, D, \cdots$, and $N-1$ with treatment $N$; and furthermore, with a coefficient of \pm 2 , in the equation corresponding to its comparison with treatment $N$. That is, $A$ will appear with a coefficient of \pm 1 in $2 n-4$ equations and with a coefficient of \pm 2 in one equation. This is true also of each of the other treatment effect constants.

Therefore, the sum of the squares of the $A$ coefficients will be $(2 n-4)+4=2 n$. This is true also of the sum of the squares of the coefficients of any other treatment effect constant. But since, as was demonstrated in the former article, on finding the sums of squares and products of the differences between the coefficients of the treatment effect constants in the original equations, one would be finding really $2 S a^{2}$, $2 S a b, \cdots, 2 S a(n-1)$ instead of $S a^{2}, S a b, \cdots, S a(n-1)$ which are the figures sought, the above value of $2 n$ must be divided by 2 , so that the value of $S a^{2}=S b^{2}=\cdots=S(n-1)^{2}=n$.

Now, in the equation corresponding to the comparison of $A$ with $B$, one of the treatment effect constants is +1 and the other -1 , so that their product is -1. In the equation corresponding to the comparison of $A$ with $N$, the coefficient of $A$ will be \pm 2 and that of $B$ will be \pm 1 , so that the products of these coefficients will be +2 . This is true also of the equation corresponding to the comparison of $B$ with $N$, where the coefficient of $B$ is \pm 2 and the coefficient of $A$ is \pm 1 . In each of the $n-3$ equations corresponding to the comparisons of each of the other treatments with $N$, the coefficients of both $A$ and $B$ will be \pm 1 , so that their product in each case will be \pm 1 . Therefore, the sum of the products of the coefficients of 
$A$ and $B$ in these equations will be $-1+2+2+(n-3)=n$. Again, this must be divided by 2 in order to get the proper value of $S a b$. Thus, $S a b=S a c=\cdots=S a(n-1)=S b c=\cdots=S(n-2)(n-1)=n / 2$.

The normal equations will be, therefore,

$$
\begin{aligned}
& A n+B n / 2+C n / 2+\cdots+(N-1) n / 2=S a y, \\
& A n / 2+B n+C n / 2+\cdots+(N-1) n / 2=S b y \text {, }
\end{aligned}
$$

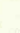

$$
\begin{aligned}
& A n / 2+B n / 2+C n / 2+\cdots+(N-1) n=S(n-1) y,
\end{aligned}
$$

giving rise to the $(n-1)$ columned determinant

$$
D_{n}=\underbrace{\left|\begin{array}{ccccc}
n & n / 2 & n / 2 & \cdots & n / 2 \\
n / 2 & n & n / 2 & \cdots & n / 2 \\
\cdot & \cdot & \cdot & & \cdot \\
\cdot & \cdot & \cdot & & \cdot \\
\cdot & \cdot & \cdot & & \cdot \\
n / 2 & n / 2 & n / 2 & \cdots & n
\end{array}\right|}_{(n-1) \text { columns }}
$$

from which the values of $C_{a a}, C_{a b}, \cdots, C_{(n-1)(n-1)}$ may be evaluated as follows.

By subtracting the last row in $D_{n}$ from each of the other rows, adding the first to the last column in the expression thus obtained, expanding the expression thus obtained by minors along the first row, and by continuing such additions of the first to the last column and expansions by minors along the first row in the resulting expressions, one obtains finally

$$
D_{n}=\left(\frac{n}{2}\right)^{n-3}\left|\begin{array}{cc}
\frac{n}{2} & 0 \\
\frac{n}{2} & n+(n-2) \frac{n}{2}
\end{array}\right|=\left(\frac{n}{2}\right)^{n-2}\left[n+(n-2) \frac{n}{2}\right]=n\left(\frac{n}{2}\right)^{n-1}
$$

Now, $C_{a a}=N_{a a} / D_{n}$, where

$$
N_{a a}=\underbrace{\left|\begin{array}{cccc}
n & n / 2 & \cdots & n / 2 \\
n / 2 & n & \cdots & n / 2 \\
\cdot & & & \\
\cdot & & & \\
n / 2 & n / 2 & \cdots & n
\end{array}\right|}_{(n-2)}
$$


The evaluation of $N_{a a}$, following a procedure identical to that used in evaluating $D_{n}$, yields the expression

$$
\begin{aligned}
& N_{a a}=\left(\frac{n}{2}\right)^{n-4}\left|\begin{array}{cc}
n / 2 & 0 \\
n / 2 & n+(n-3) \frac{n}{2}
\end{array}\right| \\
& =\left(\frac{n}{2}\right)^{n-3}\left[n+(n-3) \frac{n}{2}\right]=(n-1)\left(\frac{n}{2}\right)^{n-2} .
\end{aligned}
$$

$C_{a a}$ is, therefore, equal to $2(n-1) / n^{2}$. On finding the corresponding expressions for the other treatments, it is found that $N_{a a}=N_{b b}=\cdots=$ $N_{(n-1)(n-1)}$ and, therefore, $C_{a a}=C_{b b}=\cdots=C_{(n-1)(n-1)}=2(n-1) / n^{2}$.

Now, $C_{a b}=N_{a b} / D_{n}$, where

$$
-N_{a b}=\underbrace{\left|\begin{array}{cccc}
n / 2 & n / 2 & \cdots & n / 2 \\
n / 2 & n & \cdots & n / 2 \\
\cdot & \cdot & & \cdot \\
\cdot & \cdot & & \cdot \\
\cdot & \cdot & & \vdots \\
n / 2 & n / 2 & & n
\end{array}\right|}_{(n-2) \text { columns }}
$$

This determinant may be evaluated by subtracting the first row from each of the other ones and expanding the resulting expression by minors along the first column and continuing with expansions of the same kind successively with the resulting expressions, obtaining finally $\left(\frac{n}{2}\right)^{n-2}$. Thus, $N_{a b}=-\left(\frac{n}{2}\right)^{n-2}$, and $C_{a b}$ comes out to be $-2 / n^{2}$. The determinants from which $N_{a c}, N_{a d}, \cdots$, and $N_{(n-2)(n-1)}$ are to be evaluated will differ in every case from that used to evaluate $N_{a b}$, but by proper inversions of rows and columns they can be brought to coincide with the above one, so that $N_{a c}=N_{a d}=\cdots=N_{(n-2)(n-1)}$ and therefore, $C_{a b}=C_{a c}=\cdots=$ $C_{(n-2)(n-1)}=-2 / n^{2}$.

In experiments of this nature where each treatment is repeated $2(n-1)$ times, $N_{i i}$ is $(n-1)\left(\frac{n}{2}\right)^{n-2} 2^{n-2}=(n-1)(n)^{n-2}, N_{i j}=-\left(\frac{n}{2}\right)^{n-2} 2^{n-2}=$ $-(n)^{n-2}$, and $D_{n}=n\left(\frac{n}{2}\right)^{n-1}(2)^{n-1}=n(n)^{n-1}$. Therefore, $C_{i i}=$ $\frac{(n-1) n^{n-2}}{n(n)^{n-1}}=\frac{n-1}{n^{2}}$, and $C_{i j}=\frac{-(n)^{n-2}}{n(n)^{n-1}}=\frac{-1}{n^{2}}$. That is, as the sums of squares and products double, the values of the $C_{i i}$ 's and $C_{i j}$ 's are divided 
by 2 . If the treatments are repeated $3(n-1)$ times, $C_{i i}=\frac{1}{3}\left(\frac{2(n-1)}{n^{2}}\right)$ and $C_{i j}=\frac{1}{3}\left(\frac{-2}{n^{2}}\right)$, and similarly for other multiples of $n-1$ replications.

In the above discussion no reference has been made to $C_{n n}$ and to the $C_{i n}$ 's, that is, the values corresponding to the last variety. Now $C_{n n}=$ $C_{a a}=C_{b b}=\cdots=C_{(n-1)(n-1)}$ and $C_{i n}=C_{a b}=C_{a c}=\cdots=C_{(n-2)(n-1)}$. This may be proved as follows: $N=-(A+B+C+\cdots+(N-1))$, and therefore, for $n-1$ replications, $C_{a n}=-\left(C_{a a}+C_{a b}+C_{a c}+\cdots\right.$ $\left.+C_{a(n-1)}\right)=-\stackrel{C}{a a}_{a b}-(n-2) C_{a b}=\frac{-2(n-1)}{n^{2}}-(n-2)\left(\frac{-2}{n^{2}}\right)=\frac{-2}{n^{2}}=$ $C_{a b}=C_{a c}=\cdots=C_{(n-2)(n-1)}$. This holds also for $C_{b n}, C_{c n}, \cdots, C_{(n-1) n}$. Also, $C_{n n}=-\left(C_{a n}+C_{b n}+\cdots+C_{(n-1) n}\right)=-(n-1)\left(\frac{-2}{n^{2}}\right)=$ $2(n-1) / n^{2}=C_{a a}$, etc. The above relations kold also for other multiples of $(n-1)$ replications.

\section{Numerical example}

Diagram II shows the geographical distribution of the plots, together with the treatments and yields per plot, in cwts. per acre, of a fertilizer experiment with cotton performed by Messrs. A. Riollano and J. Pastor Rodríguez in cooperation with the author, on Coto clay at Mantilla Farm, Isabela.

Column (1) of table I shows one way in which the plots may be paired so that one may employ the procedure of calculation discussed above. As will be noticed, the paired plots lie side by side in all but two cases: where plot 56 has been paired to plot 46 , and where plot 35 has been paired to plot 47 . In these two cases the topography of the field in the section where these plots were located suggested that they should be paired. Columns (2) to:(8) show the coefficients of the treatment constants corresponding to each of the paired plots. The constant of treatment $H$ has been expressed in terms of the constants of the other seven treatments according to the relation $H=-(A+B+C+D+E+F+G)$. Column (9) shows the respective differences between the yields of the paired plots, taken in the order indicated in column (1). Though unnecessary, the plots of each pair were paired so that these differences in yields were positive. The numbers in the central portions of columns (10) to (16) are the products of the entries in columns (2) to (8) by those of column (9), as expressed at the tops of the respective columns. The first of the 2 rows at the bottom of columns (10) to (16) consists of the sums of the 
entries in the body of each of the respective columns. The entries of the second row at the bottom of these columns are each one-half of the entries of the first row and indicate the values of Say, Sby, $\cdots$, Sgy, according

\section{Diagram II}

Geographical distribution of plots of fertilizer experiment with colton

\begin{tabular}{|c|c|c|c|c|c|c|}
\hline $\begin{array}{c}52 \\
\mathrm{D} \\
5.0\end{array}$ & $\begin{array}{c}53 \\
\mathrm{~A} \\
6.1\end{array}$ & $\begin{array}{c}54 \\
\text { B } \\
6.3\end{array}$ & $\begin{array}{c}55 \\
F \\
7.6\end{array}$ & $\begin{array}{c}56 \\
\mathrm{C} \\
9.5\end{array}$ & • & \\
\hline $\begin{array}{c}48 \\
\mathrm{H} \\
4.2\end{array}$ & $\begin{array}{c}49 \\
\mathrm{C} \\
5.0\end{array}$ & $\begin{array}{c}50 \\
\mathrm{H} \\
6.6\end{array}$ & $\begin{array}{c}51 \\
\mathrm{~A} \\
5.5\end{array}$ & . & & \\
\hline $\begin{array}{c}42 \\
\text { F } \\
8.6\end{array}$ & $\begin{array}{c}43 \\
\mathrm{G} \\
6.7\end{array}$ & $\begin{array}{c}44 \\
\mathrm{E} \\
5.9\end{array}$ & $\begin{array}{c}45 \\
\mathrm{G} \\
5.2\end{array}$ & $\begin{array}{c}46 \\
\text { B } \\
8.0\end{array}$ & $\begin{array}{c}47 \\
\mathrm{D} \\
7.8\end{array}$ & \\
\hline $\begin{array}{c}36 \\
\mathrm{E} \\
7.3\end{array}$ & $\begin{array}{c}37 \\
\mathrm{H} \\
3.4\end{array}$ & $\begin{array}{c}38 \\
\mathrm{D} \\
5.5\end{array}$ & $\begin{array}{c}39 \\
F \\
7.1\end{array}$ & $\begin{array}{c}40 \\
\mathrm{E} \\
8.3\end{array}$ & $\begin{array}{c}41 \\
\mathrm{H} \\
3.5\end{array}$ & \\
\hline $\begin{array}{c}29 \\
\mathrm{C} \\
6.4\end{array}$ & $\begin{array}{c}30 \\
\mathrm{G} \\
6.4\end{array}$ & $\begin{array}{c}31 \\
\mathrm{~B} \\
7.3\end{array}$ & $\begin{array}{c}32 \\
\mathrm{C} \\
6.2\end{array}$ & $\begin{array}{c}33 \\
\mathrm{~A} \\
4.0\end{array}$ & $\begin{array}{c}34 \\
\mathrm{G} \\
8.5\end{array}$ & $\begin{array}{c}35 \\
\mathrm{~B} \\
8.4\end{array}$ \\
\hline $\begin{array}{c}22 \\
\mathrm{D} \\
7.2\end{array}$ & $\begin{array}{c}23 \\
\mathrm{~A} \\
5.3\end{array}$ & $\begin{array}{c}24 \\
\text { F } \\
6.6\end{array}$ & $\begin{array}{c}25 \\
G \\
8.3\end{array}$ & $\begin{array}{c}26 \\
\mathrm{D} \\
6.5\end{array}$ & $\begin{array}{c}27 \\
\mathrm{E} \\
8.5\end{array}$ & $\begin{array}{c}28 \\
C \\
8.7\end{array}$ \\
\hline $\begin{array}{c}15 \\
\mathrm{E} \\
6.8\end{array}$ & $\begin{array}{c}16 \\
\mathrm{~F} \\
7.2\end{array}$ & $\begin{array}{c}17 \\
\mathrm{H} \\
3.8\end{array}$ & $\begin{array}{c}18 \\
\text { B } \\
8.1\end{array}$ & $\begin{array}{c}19 \\
\mathrm{H} \\
5.5\end{array}$ & $\begin{array}{c}20 \\
\mathrm{~A} \\
5.6\end{array}$ & $\frac{21}{\mathrm{~F}}$ \\
\hline $\begin{array}{c}8 \\
\mathrm{C} \\
4.6\end{array}$ & $\begin{array}{c}9 \\
\mathrm{D} \\
7.6\end{array}$ & $\begin{array}{c}10 \\
\mathrm{~A} \\
4.0\end{array}$ & $\begin{array}{c}11 \\
\mathrm{G} \\
7.7\end{array}$ & $\begin{array}{c}12 \\
\mathrm{~F} \\
8.7\end{array}$ & $\begin{array}{c}13 \\
\text { B } \\
9.9\end{array}$ & $\begin{array}{c}14 \\
\mathrm{E} \\
7.0\end{array}$ \\
\hline $\begin{array}{c}1 \\
\text { B } \\
6.5\end{array}$ & $\begin{array}{c}2 \\
\mathrm{E} \\
5.2\end{array}$ & $\begin{array}{c}3 \\
\mathrm{G} \\
7.0\end{array}$ & $\begin{array}{c}4 \\
\mathrm{D} \\
8.1\end{array}$ & $\begin{array}{c}5 \\
\mathrm{~A} \\
5.5\end{array}$ & $\begin{array}{c}6 \\
\mathrm{C} \\
7.2\end{array}$ & $\begin{array}{c}7 \\
\mathrm{H} \\
6.0\end{array}$ \\
\hline
\end{tabular}

to whether the heading of their respective columns are $A Y, B Y, \cdots, G Y$. The theoretical reason for this procedure of finding $S a y, S b y, \cdots, S g y$ has been given elsewhere (1).

Now, in this experiment each of the 8 treatments has been paired once 


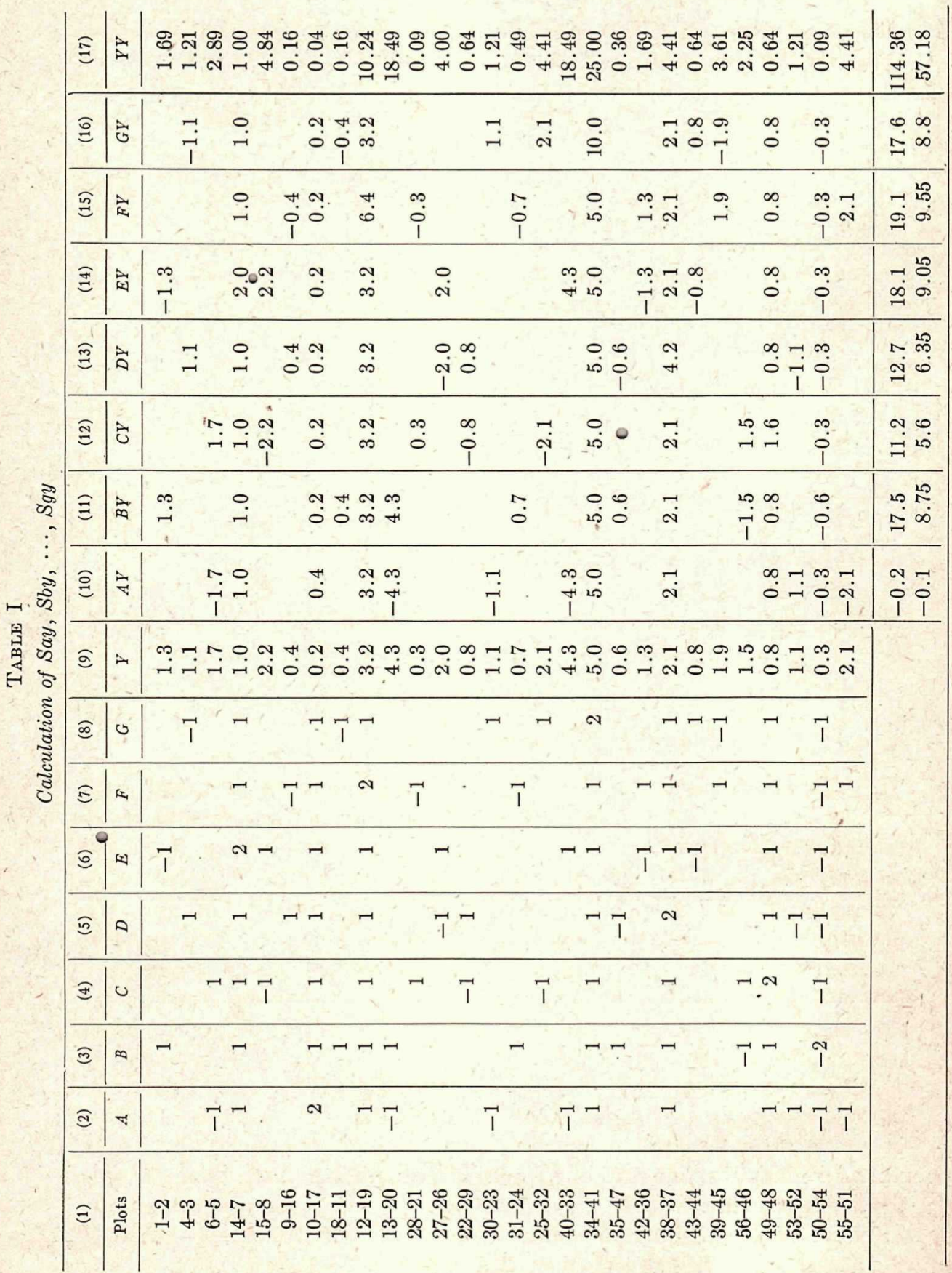


with each of the other treatments, and as mentioned in the section on the theory of the procedure of calculation,

$$
C_{a a}=C_{b b}=\cdots=C_{g g}=C_{h h}=-2(8-1) / 8^{2}=14 / 64=7 / 32,
$$

and

$C_{a b}=C_{a c}=\cdots=C_{b c}=C_{b d}=\cdots=C_{g h}=-2 / 8^{2}=-2 / 64=-1 / 32$.

Knowing, then, the $C_{i i}$ 's and the $C_{i j}$ 's, together with $S a y, S b y, \cdots, S g y$, one can calculate the yield constants corresponding to each treatment and test the significance of differences between these constants.

Thus,

$$
\begin{aligned}
& A=b_{A}=C_{a a} S a y+C_{a b} S b y+C_{a c} S c y+C_{a d} S d y \\
& +C_{a e} S e y+C_{a f} S f y+C_{a g} S g y \\
& =(7 \mathrm{Say}-\mathrm{Sby}-\mathrm{Sc \hat {y }}-\mathrm{Sdy}-\mathrm{Sey}-\mathrm{Sfy}-\mathrm{Sgy}) / 32, \\
& =(8 S a y-(S a y+S b y+S c y+S d y+S e y+S f y+S g y)) / 32 \\
& =(-0.8-48.00) / 32=-48.8 / 32=-1.525 \text {. } \\
& B=b_{B}=(8 \text { Sby }-48.00) / 32=(70.00-48.00) / 32=22 / 32=0.6875 \\
& C=b_{c}=(8 S c y-48.00) / 32=(44.80-48.00) / 32 \\
& D=b_{D}=(8 S d y-48.00) / 32=(50.80-48.00) 32=2.8 / 32=0.0875 \\
& E=b_{E}=(8 \text { Sey }-48.00) / 32=(72.40-48.00) / 32=24.4 / 32=0.7625 \\
& F=b_{F}=(8 S f y-48.00) / 32=(76.40-48.00) / 32=28.4 / 32=0.8875 \\
& G=b_{G}=(8 S g y-48.00) / 32=(70.40-48.00) / 32=22.4 / 32=0.7000 \\
& H=-(A+B+C+D+E+F+G)=-1.5000 \text {. }
\end{aligned}
$$

To make comparisons between these statistics, one must calculate their standard errors. The sum of the squared deviations of the yields corrected for differences in fertility between the different 2-plot blocks is found by dividing the sum of the squares of the entries in column (9) of table I by 2 . The squares of the corresponding entries constitute the body of column (17) of table I, their sum is the next to the last item in that column, and the required sum of squared deviations corrected for differences in fertility between the 2-plot blocks is one-half the next to the last item in column (17), that is, 57.18. This sum of squared deviations is subject to 28 degrees of freedom, since 28 degrees of freedom have been lost: one $d f$ corresponding to the mean of all the yields and $27 d f$ due to the fitting of the 27 different block constants necessary to express the relations between the fertilities of the 28 2-plot blocks.

The reduction of the sum of squared deviations due to the fitting of the treatment constants to the yield data is, according to the usual relation, $S y^{\prime} y^{\prime}=A S a y+B S b y+\cdots+G S g y=(-1.525)(-0.1)+0.6875$ $(8.75)+\cdots+0.7000(8.80)=27.70$, subject to $7 d f$. 
The reduced sum of squared deviations is, therefore, $S\left(Y-Y^{\prime}\right)^{2}=$ $S y y-S y^{\prime} y^{\prime}=57.18-27.70=29.48$, subject to $28-7=21 d f$. The estimate of the reduced variance, $V^{\prime}$, is then $29.48 / 21=1.4037$.

The reduction in the sum of squared deviations due to the use of the 7 treatment constants may be tested by means of the $F$-test as follows: $F=27.70 / 7(1.4037)=27.70 / 9.8259=2.82$. Since the value of $F$ at the $5 \%$ point, for 7 and $21 d f$ is 2.49 , the reduction in the sum of squared deviations due to the fitting of the treatment constants is a significant one.

The total sura of squared deviations of the yields of this experiment is 134.29 , and as found above, the sum of squared deviations of the yields corrected for differences in fertility between blocks is 57.18. The fitting of the block constants led therefore to a reduction of $134.29-57.18=$ 77.11 in the sum of squared deviations. These relations are indicated in table II.

TABLE II

Analysis of the total sum of squared deviations following the 2-plot block method

\begin{tabular}{|c|c|c|c|c|c|c|}
\hline \multirow{2}{*}{ Source of the deviations } & \multirow{2}{*}{$\begin{array}{c}\text { Degrees } \\
\text { of freedom }\end{array}$} & \multirow{2}{*}{$\begin{array}{c}\begin{array}{c}\text { Sum of } \\
\text { squared } \\
\text { deviations }\end{array}\end{array}$} & \multirow{2}{*}{$\begin{array}{l}\text { Variance } \\
\text { estimate }\end{array}$} & \multicolumn{3}{|c|}{$F$ values } \\
\hline & & & & $\begin{array}{l}\text { Experi- } \\
\text { mental }\end{array}$ & $5 \%$ & $1 \%$ \\
\hline Total. . & 55 & 134.29 & & & & \\
\hline Blocks......... & 27 & 77.11 & & & & \\
\hline Treatments... & 7 & 29.48 & 4.21 & 2.82 & 2.49 & 3.65 \\
\hline Error......... & 21 & 27.70 & 1.4037 & & & \\
\hline
\end{tabular}

In testing by means of the $t$-test any difference between the fitted treatment constants, the variance of any such difference would be found by multiplying $V^{\prime}$ by the corresponding factor of the nature of $C_{i i}-2 C_{i j}+$ $C_{j j}$. Since, in the case of this experiment all the $C_{i i}$ 's are equal and all the $C_{i j}$ 's are equal, the variance of the difference between any two treatment constants is $(7 / 32-2(-1 / 32)+7 / 32) 1.4037=0.70185$. The standard error of any such difference is, therefore, $(0.70185)^{1 / 2}=0.8378$, and any such difference, to be significant at the $5 \%$ point, must exceed $2.080(0.8378)=1.74$ cwts. cotton per acre.

The treatments compared in this test are described in table III. The corrected mean yield of any given treatment is found by adding algebraically the respective treatment yield constant as found above to the mean yield of all plots, 6.64 .

Since treatments $E, F$, and $G$ differed only with respect to the rate of the $\mathrm{K}_{2} \mathrm{O}$ applications, the comparisons of their corrected mean yields or, what is equivalent, of their treatment yields constants, will furnish information on the effect of the $\mathrm{K}_{2} \mathrm{O}$ applications on the cotton yields. Such 
comparisons indicate that the effect of the $\mathrm{K}_{2} \mathrm{O}$ applications on the cotton yields was not significant since the largest difference between any two of said constants was only 0.1875 , whereas such a difference would have to exceed $1.74 \mathrm{cwts}$ /A. to be significant at the $5 \%$ point.

Treatments $C, D$, and $G$ varied only with respect to the $\mathrm{P}_{2} \mathrm{O}_{5}$ applications. In this case also, none of the differences between the yield constants were significant although they were of a larger magnitude than in the case of the $\mathrm{K}_{2} \mathrm{O}$ applications.

A similar comparison of the yield constants of treatments $A, B$, and $G$ indicates that there was a significant increase in yield, caiused by the application of at least $100 \mathrm{lbs} . \mathrm{NH}_{3}$ per acre, since the difference between the yield constants of treatments $A$ and $B$ was 2.22 cwts. cotton per acre, and the corresponding difference between the yield constants of treatments

TABLE III

Description of treatments and corrected mean yields

\begin{tabular}{|c|c|c|c|c|c|}
\hline \multirow{2}{*}{ Treatment letter } & \multicolumn{3}{|c|}{ Lbs. of nutrient applied per acre } & \multirow{2}{*}{$\begin{array}{c}\text { Treatment } \\
\text { yield constant }\end{array}$} & \multirow{2}{*}{$\begin{array}{l}\text { Corrected } \\
\text { mean yield } \\
\text { (cwts./acre) }\end{array}$} \\
\hline & $\mathrm{NH}_{3}$ & $\mathrm{P}_{2} \mathrm{O}_{5}$ & $\mathrm{~K}_{2} \mathrm{O}$ & & \\
\hline A & 0 & 200 & 200 & -1.5250 & 5.11 \\
\hline B & 100 & 200 & 200 & 0.6875 & 7.33 \\
\hline $\mathrm{C}$ & 200 & 0 & 200 & -0.1000 & 6.54 \\
\hline D & 200 & 100 & 200 & 0.0875 & 6.73 \\
\hline $\mathrm{E}$ & 200 & 200 & 0 & 0.7625 & 7.40 \\
\hline $\mathrm{F}$ & . 200 & 200 & 100 & 0.8875 & 7.53 \\
\hline G & 200 & 200 & 200 & 0.7000 & 7.34 \\
\hline $\mathrm{H}$ & 0 & 0 & 0 & -1.5000 & 5.14 \\
\hline
\end{tabular}

$A$ and $G$ was 2.23 cwts. cotton per acre, both differences being significant at the $5 \%$ point and nearly so at the $1 \%$ point. The difference between the yield constants of treatments $B$ and $G$ was not significant indicating that the second $100 \mathrm{lbs}$. $\mathrm{NH}_{3}$ applied per acre did not affect significantly the yields already obtained with the first $100 \mathrm{lbs}$. $\mathrm{NH}_{3}$ applied per acre.

The yield constant of treatment $H$ was almost equal to that of treatment $A$, corroborating the conclusions drawn above that nitrogen was the only one of the three elements tested which affected the yields significantly.

This experiment might have also been interpreted by using only 3 constants to explain the variations in yield caused by the various treatments as described in the article (1) previously referred to. Such a study was made yielding the same results previously obtained, except that the partial regression coefficient of the yields on the nitrogen applications came out to be significant at the $1 \%$ point. 
34 JOURNAL OF AGRICULTURE OF UNIVERSITY OF PUERTO RICO

\section{SUMMARY}

A new method of performing field experiments with relatively small numbers of treatments is described. The requirement to be fulfilled by the layouts of such field tests is specified and examples of possible designs for a 5 -treatment experiment are illustrated. The theory of the procedure of calculation is discussed and a numerical example of said calculations is furnished in connection with the interpretation of a fertilizer experiment performed with cotton.

\section{REFERENCE}

1. Capó, B. G. A method of interpreting the results of field trials. This Journal: 28 (1), 7-21. 1944. 